\title{
Modeling of ammonothermal growth processes of GaN crystal in large-size pressure systems
}

\author{
Qi-Sheng Chen · Yan-Ni Jiang · Jun-Yi Yan • \\ Wei Li $\cdot$ V. Prasad
}

Received: 25 July 2010/ Accepted: 1 December 2010/Published online: 11 February 2011

(C) Springer Science+Business Media B.V. 2011

\begin{abstract}
Gallium nitride $(\mathrm{GaN})$ is a wide-bandgap semiconductor material with a wide array of applications in optoelectronics and electronics. Modeling of the fluid flow and thermal fields is discussed, and simulations of velocity and volumetricflow-rate profiles in different pressure systems are shown. The nutrient is considered as a porous media bed, and the flow is simulated using the Darcy-BrinkmanForchheimer model. The resulting governing equations are solved using the finitevolume method. We analyzed the heat and mass transfer behaviors in autoclaves with diameters of 2.22, 4.44, and $10 \mathrm{~cm}$. The effects of baffle design on flow pattern, and heat and mass transfer in different autoclaves are analyzed. For the research-grade autoclave with diameter of $2.22 \mathrm{~cm}$, the constraint for the $\mathrm{GaN}$ growth is found to be the growth kinetics and the total area of seed surfaces in the case of baffle opening of $10 \%$. For large-size pressure systems, the concentration profiles change dramatically due to stronger convection at higher Grashof numbers. The volumetric flow rates of the solvent across the baffles are calculated. Since ammonothermal growth experiments are expensive and time consuming, modeling becomes an effective tool for research and optimization of ammonothermal growth processes.
\end{abstract}

Keywords GaN · Ammonothermal growth · Baffle opening · Fluid flow · Thermal fields

Q.-S. Chen $(\bowtie) \cdot$ Y.-N. Jiang · J.-Y. Yan · W. Li

Institute of Mechanics, Chinese Academy of Sciences, 15 Bei Si Huan Xi Road,

Beijing 100190, China

e-mail: qschen@imech.ac.cn

V. Prasad

University of North Texas, PO Box 310979, Denton, TX 76203-0979, USA 


\section{Introduction}

Gallium nitride $(\mathrm{GaN})$ is a wide-bandgap semiconductor with a wide array of applications in optoelectronics and electronics. GaN devices have shown rapid development in the last 10 years since the major technological hurdles were overcome in the early 1990s. The similarity of ammonia and water as polar solvents allows $\mathrm{GaN}$ crystals to grow in ammonia solvents in the same way as in hydrothermal growth of oxide crystals in high-pressure water solutions.

Ammonothermal growth of 1-inch-size (0001) GaN crystal in a cylindrical highpressure autoclave with internal diameter of $40 \mathrm{~mm}$ was reported by Hashimoto et al. [1-3]. Basic mineralizer was used to obtain retrograde solubility of $\mathrm{GaN}$ in supercritical ammonobasic solutions. The nutrient was placed in the colder (upper) region, and free-standing $c$-plane hydride vapor-phase epitaxy (HVPE) GaN seed crystals were placed in the hotter (lower) region. Temperatures on the outer surface of the autoclave were set at $675{ }^{\circ} \mathrm{C}$ for the lower zone and $625^{\circ} \mathrm{C}$ for the upper zone, and the pressure was about $214 \mathrm{MPa}$. Metallic Ga was used as a nutrient, and $1 \mathrm{M} \mathrm{NaNH}_{2}$ and $0.05 \mathrm{M} \mathrm{NaI}$ were used as mineralizers. About 15 - $\mu \mathrm{m}$-thick GaN films were uniformly grown on each side of the seed. The growth rate was about $0.02 \mathrm{~mm} /$ day. The Ga-polar surface was filled with pits, whereas the N-polar surface was featureless. Photoluminescence (PL) characterization also indicated qualitatively uniform optical properties on each side of the crystal.

D'Evelyn et al. [4] gave an overview of the high-pressure ammonothermal method, which is based on adaptation of high-pressure apparatus developed for diamond growth. They described recent progress in reduction of impurity concentrations, wafering, and fabrication of homoepitaxial laser diodes on substrates. Crystals were grown at temperatures between 600 and $1000{ }^{\circ} \mathrm{C}$ at liquid ammonia fills of about $70-95 \%$ in volume. The pressure is estimated as lying between about 5 and $200 \mathrm{MPa}$.

Dwilinski et al. [5, 6] grew 1-inch GaN crystals using basic mineralizers. The basic mineralizers provide a nondestructive environment for the autoclave materials (Ni-Cr alloys), in contrast to acidic mineralizers which require use of Pt liners. GaN crystals obtained using basic mineralizers are of pure hexagonal phase. The solubilities of $\mathrm{GaN}$ in the $\mathrm{NH}_{3}+\mathrm{KNH}_{2}$ solution with molar ratio $\mathrm{KNH}_{2}: \mathrm{NH}_{3}=$ 0.07 were measured as a function of pressure at two temperatures: 400 and $500{ }^{\circ} \mathrm{C}$. The maximum solubility of $\mathrm{GaN}$ was about $3 \mathrm{~mol} \%$.

Fukuda and Ehrentraut [7-9] grew 0.5-mm-thick GaN on a 1-inch HVPE seed at growth temperature of about $500{ }^{\circ} \mathrm{C}$ and pressure of less than $130 \mathrm{MPa}$. The growth rate was about $0.04 \mathrm{~mm} / \mathrm{day}$. Ehrentraut et al. [10] reviewed the development in acid ammonothermal crystal growth of GaN. They grew 0.5 -mm-thick $\mathrm{GaN}$ on a 2-inch HVPE GaN seed in an autoclave of $100 \mathrm{~mm}$ inner diameter. The growth temperatures were $425 / 525{ }^{\circ} \mathrm{C}$ for the upper/lower zones, and pressure was $145 \mathrm{MPa}$. They estimated that the growth rate can reach about $1 \mathrm{~mm} /$ day for $1 \mathrm{~mol} \%$ mineralizer of $\mathrm{NH}_{4} \mathrm{Cl}$ in $\mathrm{NH}_{3}$, when the solubility of $\mathrm{GaN}$ is about $20 \mathrm{~mol} \%$ in $\mathrm{NH}_{3}$.

Chen et al. [11] studied the effects of particle size on flow pattern and temperature distribution in the autoclave. The autoclave used had internal diameter of $0.932 \mathrm{~cm}$ and external diameter of $3.5 \mathrm{~cm}$ (Tem-Press MRA 138R, volume $12.5 \mathrm{ml}$ ). Chen et al. [12] modeled the GaN growth process in an autoclave with 
internal diameter of $2.22 \mathrm{~cm}$ and external diameter of $7.62 \mathrm{~cm}$ (Tem-Press MRA $378 \mathrm{R}$, volume $134 \mathrm{ml}$ ). Flow and temperature patterns inside the autoclave with baffle openings of $15 \%$ and $20 \%$ in cross-sectional area were analyzed. Numerical studies were also performed for an ammonothermal system with retrograde solubility [13]. The charge of $10.1 \mathrm{~cm}$ height is put $2.5 \mathrm{~cm}$ above the baffle. Herein, the effect of baffle design on the fluid flow and heat and mass transfer in large-size pressure systems will be studied.

\section{Physical and mathematical models}

The ammonothermal growth process uses ammonia solvents under high temperatures and high pressures to dissolve and recrystallize reagents. After the system is pressurized, solvent such as ammonia occupies most of the volume. The convection system for ammonothermal growth consists of a porous bed whose height changes with the growth, a fluid layer overlying this porous bed, a metal baffle with holes which lies above the porous bed, and a solid seed plates whose size increases with the growth. Figure 1 shows a schematic of a growth system that has been used to synthesize GaN. The autoclave has internal diameter of $2.22 \mathrm{~cm}$, external diameter of $7.62 \mathrm{~cm}$, internal height of $35.56 \mathrm{~cm}$, and external height of $38.10 \mathrm{~cm}$ (TemPress MRA 378R, volume $134 \mathrm{ml}$ ). The thickness of the sidewall of the autoclave is $2.54 \mathrm{~cm}$. The baffle is located at a distance of $15.24 \mathrm{~cm}$ from the bottom of the autoclave. A baffle made from $0.28 \mathrm{~mm} \mathrm{Ag}$ foil is used to divide the autoclave into two parts: the upper and lower portions.

Hence, the upper portion can be considered as a fluid layer with the assumption of incompressible flow and the Boussinesq approximation [14, 15], and the NavierStokes equations can be used in the fluid layer. Suppose the density has a linear temperature dependence of the form $\rho=\rho_{0}\left[1-\beta\left(T-T_{0}\right)\right]$, where $\rho, T$, and $\beta$ are density, temperature, and isobaric coefficient of expansion, and $\rho_{0}$ and $T_{0}$ are constant reference values for the density and temperature, respectively. In the solid region which comprises the autoclave walls, the baffle, and the seeds, only conductive heat transfer is considered. In the fluid region, convective heat transfer is considered.

Fig. 1 Schematic of an ammonothermal growth system

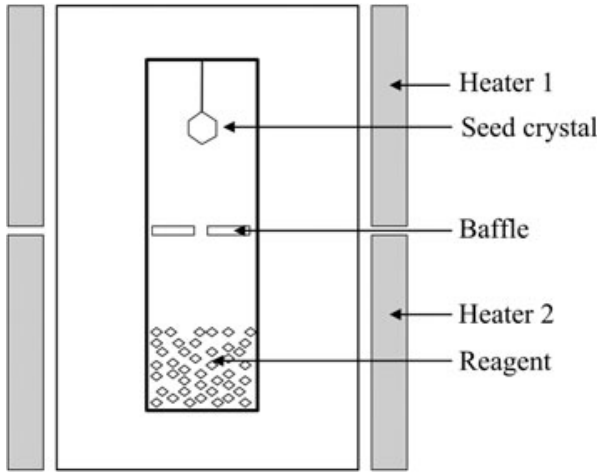


The nutrient particles of $\mathrm{GaN}$ in the bottom of the autoclave can be considered as a porous medium. In this case, the Darcy-Brinkman-Forchheimer model can be employed in the porous layer [15]. The dimensionless parameters of the system are listed as follows:

$$
A=H / R, \mathrm{Gr}=g \beta R^{3} \Delta T / v^{2}, \operatorname{Pr}=v / \alpha, S c=v / D, \mathrm{Da}=K / R^{2}, \mathrm{Fs}=b / R
$$

where $A$, Gr, Pr, Sc, Da, and Fs denote the aspect ratio, Grashof number, Prandtl number, Schmidt number, Darcy number, and Forchheimer number, respectively. $H$ is the internal height of the autoclave, $R$ is the internal radius of the autoclave, $g$ is acceleration due to gravity, $\Delta T$ is the maximum temperature difference on the sidewall of the autoclave, $v$ is kinematic viscosity, $\alpha$ is thermal diffusivity, $D$ is the mass diffusivity, the permeability of porous matrix $K=\frac{d_{\mathrm{p}}^{2} \varepsilon^{3}}{150(1-\varepsilon)^{2}}$ with $d_{\mathrm{p}}$ as the average diameter of the nutrient particles, and the Forchheimer coefficient $b=\frac{1.75}{\sqrt{150} \varepsilon^{1.5}} K^{0.5}$.

The governing equations in the porous and fluid layers can be combined by defining a binary parameter $B$ as: $B=0$ in the fluid layer and $B=1$ in the porous layer. The porosity $\varepsilon=0$ in solid, $0<\varepsilon<1$ in porous layer, and $\varepsilon=1$ in fluid layer. The combined governing equations in a cylindrical coordinate system are

$$
\begin{gathered}
\frac{\partial\left(\varepsilon \rho_{\mathrm{f}}\right)}{\partial t}+\nabla \cdot\left(\rho_{\mathrm{f}} \mathbf{u}\right)=0, \\
\frac{\partial}{\partial t}\left(\varepsilon \rho_{\mathrm{f}} \mathbf{u}\right)+\rho_{\mathrm{f}}(\mathbf{u} \cdot \nabla) \mathbf{u}=-\varepsilon^{2} \nabla p-\rho_{\mathrm{f}} \varepsilon^{2} \beta\left(T-T_{0}\right) \mathbf{g}+\varepsilon^{2} \nabla \cdot\left(\mu_{\mathrm{e}} \nabla \mathbf{u}\right) \\
-B \varepsilon^{2}\left[\left(\frac{\mu_{\mathrm{f}}}{K}+\frac{\rho_{\mathrm{f}} b}{K}|\mathbf{u}|\right) \mathbf{u}\right], \\
\left(\rho c_{\mathrm{p}}\right)_{\mathrm{e}} \frac{\partial T}{\partial t}+\left(\rho c_{\mathrm{p}}\right)_{\mathrm{f}}[(\mathbf{u} \cdot \nabla) T]=\nabla \cdot\left(k_{\mathrm{e}} \nabla T\right), \\
\frac{\partial}{\partial t}\left(\rho_{\mathrm{e}} C\right)+\nabla \cdot\left(\rho_{\mathrm{e}} \mathbf{u} C\right)=\rho_{\mathrm{e}} D_{\mathrm{e}} \nabla^{2} C-B \rho_{\mathrm{e}} k_{\mathrm{g}} \beta_{\mathrm{s}} \varepsilon\left(C-C_{0}\right),
\end{gathered}
$$

where $\mu, k$, and $c_{\mathrm{p}}$ denote dynamic viscosity, thermal conductivity, and specific heat, respectively. $\mathbf{g}$ is the gravity vector, and $k_{\mathrm{g}}$ is the mass transfer coefficient for crystal growth from solution at the growth interface. $\beta_{\mathrm{s}}$ is the surface area to volume ratio. Subscripts "f" and " $\mathrm{f}$ " denote fluid and effective, respectively. The reaction and the growth rate expression at the growth interface are as follows:

$$
\begin{gathered}
\mathrm{GaN}+(x-3) \mathrm{NH}_{3}+2 \mathrm{NH}_{3} \leftrightarrow \mathrm{Ga}\left(\mathrm{NH}_{2}\right)_{x}, \\
v_{\mathrm{c}}=\frac{D_{\mathrm{e}} \quad \partial\left(\rho_{\mathrm{f}} c\right)}{\left(\rho_{\mathrm{S}}-\rho_{\mathrm{f}} c\right) \quad \partial n}=k_{\mathrm{g}}\left(c_{i}-c_{0}\right),
\end{gathered}
$$

where the mass transfer coefficient for crystal growth, $k_{\mathrm{g}} \approx 10^{-6} \mathrm{~m} / \mathrm{s}$.

The following scales are used to nondimensionalize the governing equations: length $R$; velocity, $u_{0}=v / R$; time, $t_{0}=R^{2} / v$; pressure, $\rho v^{2} / R^{2}$; and temperature, $T_{\mathrm{H}}-T_{\mathrm{L}} . T_{\mathrm{H}}$ and $T_{\mathrm{L}}$ are the high temperature and low temperature applied on the 
sidewall of the autoclave, respectively. The resulting nondimensionalized equations are

$$
\begin{gathered}
\frac{\partial \varepsilon \bar{\rho}}{\partial t}+\frac{1}{r} \frac{\partial}{\partial r}(r \bar{\rho} u)+\frac{\partial}{\partial z}(\bar{\rho} w)=0, \\
\frac{\partial}{\partial t}(\varepsilon \bar{\rho} u)+\frac{1}{r} \frac{\partial}{\partial r}(r \bar{\rho} u u)+\frac{\partial}{\partial z}(\bar{\rho} w u)=\bar{\mu} \varepsilon^{2}\left[\frac{1}{r} \frac{\partial}{\partial r}\left(r \frac{\partial u}{\partial r}\right)+\frac{\partial^{2} u}{\partial z^{2}}-\frac{u}{r^{2}}\right]-\varepsilon^{2} \frac{\partial p}{\partial r} \\
-B \varepsilon^{2}\left(\frac{1}{\operatorname{Re} D a}+\frac{F s}{D a}|\mathbf{u}|\right) \bar{\rho} u, \\
\frac{\partial}{\partial t}(\varepsilon \bar{\rho} w)+\frac{1}{r} \frac{\partial}{\partial r}(r \bar{\rho} u w)+\frac{\partial}{\partial z}(\bar{\rho} w w)=\bar{\mu} \varepsilon^{2}\left[\frac{1}{r} \frac{\partial}{\partial r}\left(r \frac{\partial w}{\partial r}\right)+\frac{\partial^{2} w}{\partial z^{2}}\right]-\varepsilon^{2} \frac{\partial p}{\partial z}+\varepsilon^{2} G r \Theta \\
-B \varepsilon^{2}\left(\frac{1}{\operatorname{Re} D a}+\frac{F s}{D a}|\mathbf{u}|\right) \bar{\rho} w, \\
\bar{\rho} \bar{c}_{\mathrm{p}} \frac{\partial \Theta}{\partial t}+\frac{1}{r} \frac{\partial}{\partial r}(r \bar{\rho} u \Theta)+\frac{\partial}{\partial z}(\bar{\rho} w \Theta)=\frac{1}{\operatorname{Pr}} \bar{k}\left[\frac{1}{r} \frac{\partial}{\partial r}\left(r \frac{\partial \Theta}{\partial r}\right)+\frac{\partial}{\partial z}\left(\frac{\partial \Theta}{\partial z}\right)\right], \\
\bar{\rho} \frac{\partial c}{\partial t}+\frac{1}{r} \frac{\partial}{\partial r}(r \bar{\rho} u c)+\frac{\partial}{\partial z}(\bar{\rho} w c)=\frac{1}{S c} \bar{D}\left[\frac{1}{r} \frac{\partial}{\partial r}\left(r \frac{\partial c}{\partial r}\right)+\frac{\partial}{\partial z}\left(\frac{\partial c}{\partial z}\right)\right],
\end{gathered}
$$

where $\bar{\rho}=\frac{\rho_{\mathrm{e}}}{\rho_{\mathrm{f}}}, \bar{\mu}=\frac{\mu_{\mathrm{e}}}{\mu_{\mathrm{f}}}, \bar{c}_{\mathrm{p}}=\frac{c_{\mathrm{pe}}}{c_{\mathrm{pf}}}, \bar{k}=\frac{k_{\mathrm{e}}}{k_{\mathrm{f}}}$, and $\bar{D}=\frac{D_{\mathrm{e}}}{D_{\mathrm{f}}}$.

\section{Results and discussion}

The ammonothermal growth system with forward solubility is considered, in which case the charge is put below the baffle. Figure 2 shows the temperature distribution

Fig. 2 Temperature field in an ammonothermal system with inner diameter of $2.22 \mathrm{~cm}$ and baffle opening of $10 \%$ in the cross-sectional area (central opening of $5 \%$ and ring opening of $5 \%$ )

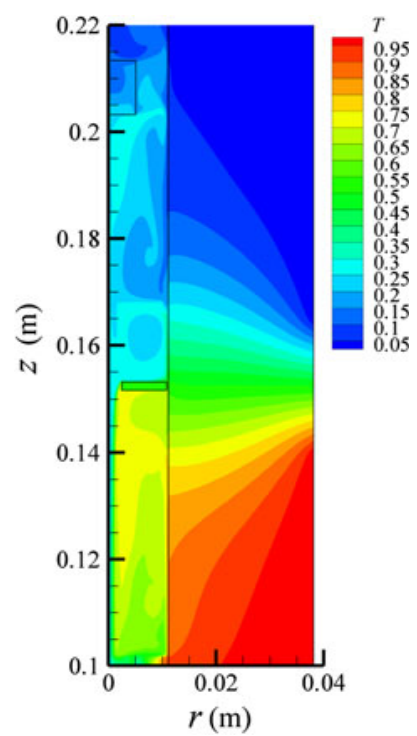


Fig. 3 Fluid flow in the system with baffle opening of $10 \%$

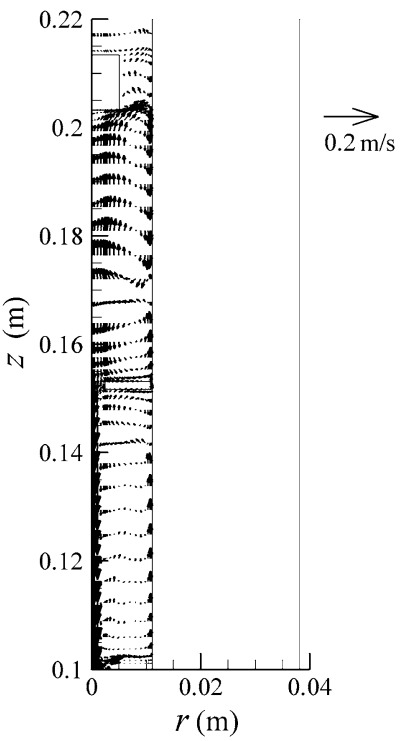

in the autoclave with inner diameter of $2.22 \mathrm{~cm}$. The temperature gradients across the baffle are large, which may cause depositions of GaN near the baffle. The baffle opening is selected to achieve a certain temperature difference across the baffle, and thus certain supersaturation in the growth zone of the autoclave and growth rate of crystals can be achieved. The upward flow in the central hole can bring hot solvent towards the bottom of the seed (Fig. 3). The flow strength in the fluid layer depends on the Grashof number, which is proportional to the temperature difference on the sidewall and the cube of the internal radius of the autoclave. The dimensionless parameters used are: $\operatorname{Pr}=0.73, \mathrm{Gr}=6.0 \times 10^{7}, \mathrm{Da}=3.8 \times 10^{-6}$, and $\mathrm{Fs}=$ $1.1 \times 10^{-3}$. Flow in the charge layer depends on the modified Grashof number, i.e., the product of the Grashof number and the Darcy number, which is proportional to the square of the average diameter of particles. The flow strength in the porous layer can be increased by increasing the size of particles, or by putting particles in bundles as in the hydrothermal growth. Reducing the baffle opening can reduce the overall flow strength in the fluid region and the volumetric flow rate through the central hole of the baffle.

The concentration field is shown in Fig. 4. The constraints for the ammonothermal growth include dissolving of charge, nucleation on the sidewall, transfer of nutrient from charge to seed, and growth kinetics. The upward flow through the central hole can bring the hot solvent below the baffle to the bottom of the seed, resulting in nonuniform temperature distributions on the seed surfaces. The temperature decreases from the bottom of the seed to the side of the seed, and deposition may occur on the side and top of the seed. The mass transfer between the dissolving zone and the growth zone is important for the continued growth of $\mathrm{GaN}$ crystals. From the calculation, the total deposition rate including deposition on the seeds and sidewalls of the autoclave is about $9 \mathrm{~g} / \mathrm{day}$, and the growth rate is about 
Fig. 4 Concentration field in the system with inner diameter of $2.22 \mathrm{~cm}$ and baffle opening of $10 \%$

Fig. 5 Average velocity in the central hole and ring gap in the system with inner diameter of $2.22 \mathrm{~cm}$ and baffle opening of $10 \%$
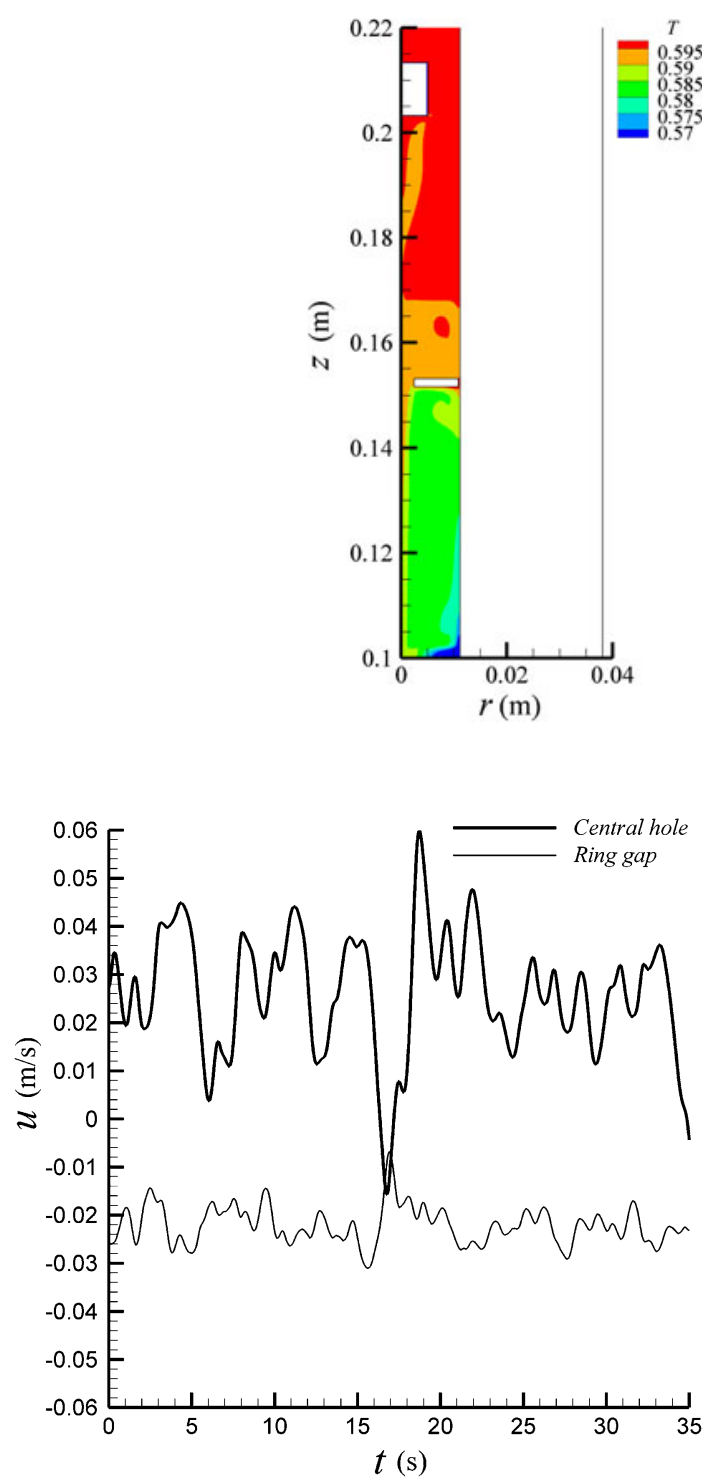

$1 \mathrm{~mm} /$ day if the mass transfer coefficient for crystal growth is taken as $10^{-6} \mathrm{~m} / \mathrm{s}$ and the charge particle size is $1 \mathrm{~mm}$. For particle size of $1 \mathrm{~mm}$, the dimensionless concentration inside the autoclave is much less than 1, and the low supersaturation in the growth zone can reduce depositions on the baffle and the sidewall of the autoclave.

Figures 5, 6 and 7 show the evolving average flow velocity in the center hole and the ring gap for systems with different inner diameters. For the system with inner diameter of $2.22 \mathrm{~cm}$ and baffle opening of $10 \%$ in the cross-sectional area (center opening of 5\% and ring opening of 5\%), the average velocity in the central hole is 
Fig. 6 Average velocity in the central hole and ring gap in the system with inner diameter of $4.44 \mathrm{~cm}$ and baffle opening of $10 \%$
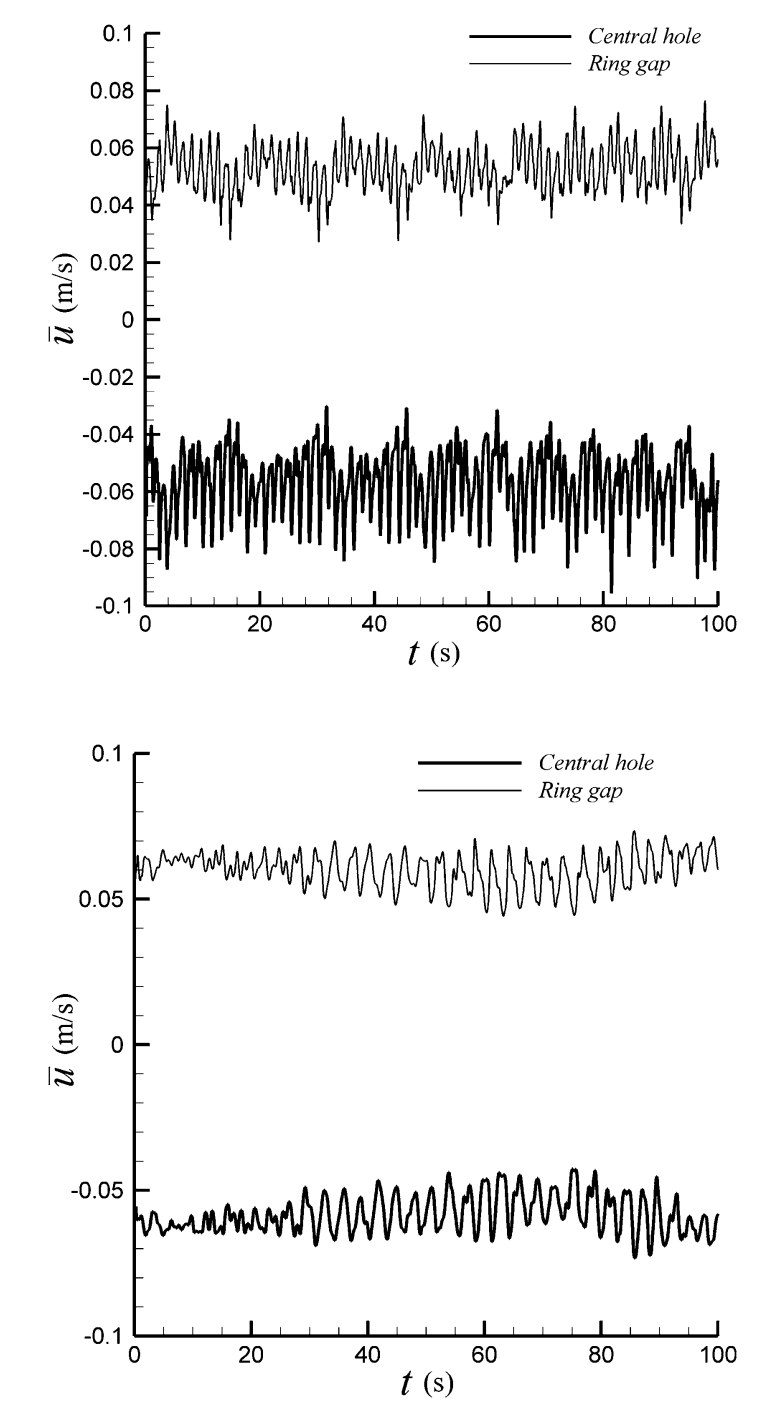

Fig. 7 Average velocity in the central hole and ring gap in the system with inner diameter of $10 \mathrm{~cm}$ and baffle opening of $10 \%$

about $0.02 \mathrm{~m} / \mathrm{s}$, while the average velocity in the ring gap is about $-0.02 \mathrm{~m} / \mathrm{s}$. The fluctuation of velocity in the central hole is lager than that in the ring gap. The average volumetric flow rate is about $4 \times 10^{-7} \mathrm{~m}^{3} / \mathrm{s}$.

For $70 \%$ fill, the density of the ammonia under supercritical conditions is about $476 \mathrm{~kg} / \mathrm{m}^{3}$, while the density of the ammonia before filling is taken as $681 \mathrm{~kg} / \mathrm{m}^{3}$ at $-33^{\circ} \mathrm{C}$. From the calculation, the average volumetric flow rate is about $4 \times 10^{-7} \mathrm{~m}^{3} / \mathrm{s}$ (Fig. 8), and the transport rate of nutrient through the central hole of the baffle is about $490 \mathrm{~g} /$ day if solubility is taken as $3 \%$ by weight. The transport rate of nutrient through the central hole is much larger than the deposition rate of $\mathrm{GaN}$ in the growth zone. So, the constraint for continued growth of $\mathrm{GaN}$ is the growth kinetics and the total area of seed surfaces in the case of $10 \%$ baffle opening. 
Fig. 8 Average volumetric flow rate in the central hole and ring gap in the system with inner diameter of $2.22 \mathrm{~cm}$ and baffle opening of $10 \%$
Fig. 9 Average volumetric flow rate in the central hole and ring gap in the system with inner diameter of $4.44 \mathrm{~cm}$ and baffle opening of $10 \%$
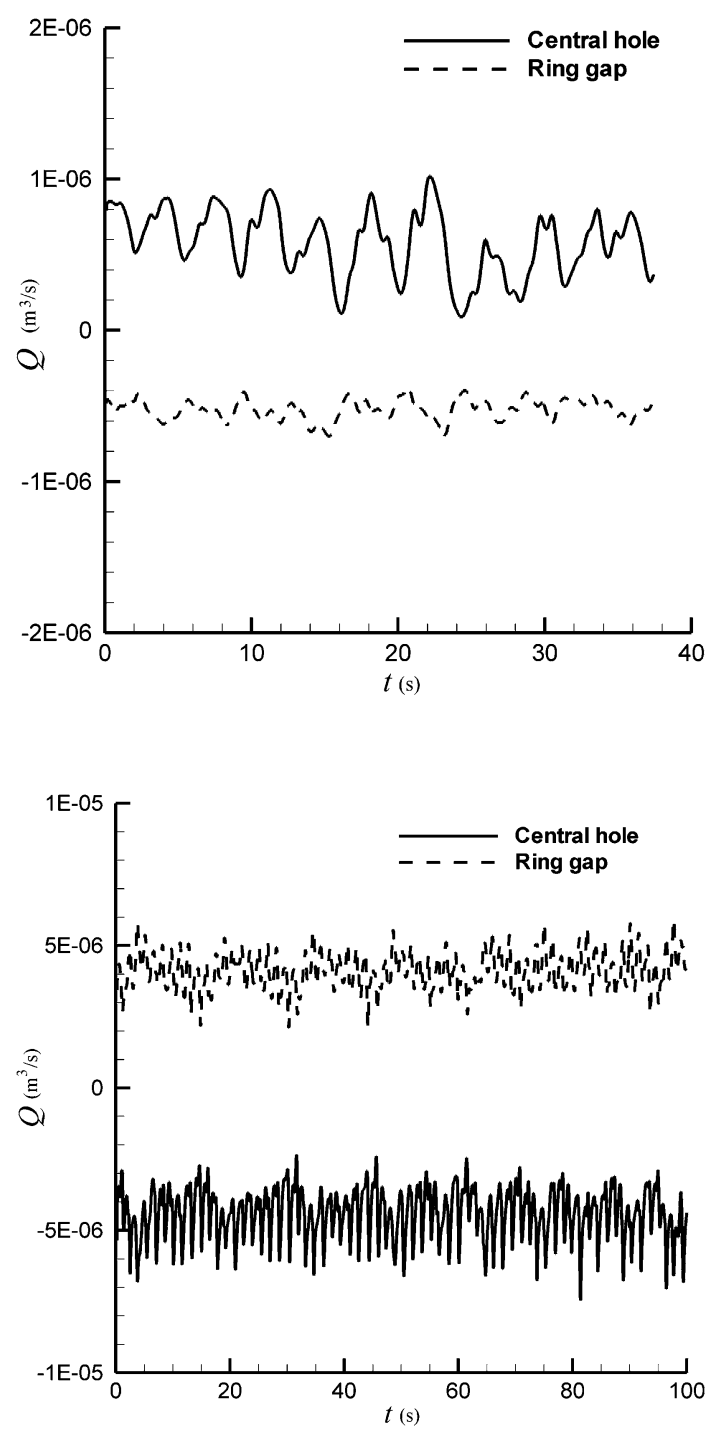

For the system with inner diameter of $4.44 \mathrm{~cm}$ and baffle opening of $10 \%$ in the cross-sectional area (center opening of 5\% and ring opening of 5\%), the flow is cyclic with time (Fig. 6). The average velocity is about $-0.05 \mathrm{~m} / \mathrm{s}$ in the central hole, and the average velocity is about $0.05 \mathrm{~m} / \mathrm{s}$ in the ring gap. The average volumetric flow rate is about $4 \times 10^{-6} \mathrm{~m}^{3} / \mathrm{s}$ (Fig. 9). When the inner diameter increases from 2.22 to $4.44 \mathrm{~cm}$, the volumetric flow rate through the central hole increases almost 9 times.

For the system with inner diameter of $10 \mathrm{~cm}$ and baffle opening of $10 \%$ in the cross-sectional area (center opening of 5\% and ring opening of 5\%), the fluid flow is a cyclic flow around the baffle. The average velocity is about $-0.06 \mathrm{~m} / \mathrm{s}$ in the 
Fig. 10 Average volumetric flow rate in the central hole and ring gap in the system with inner diameter of $10 \mathrm{~cm}$ and baffle opening of $10 \%$

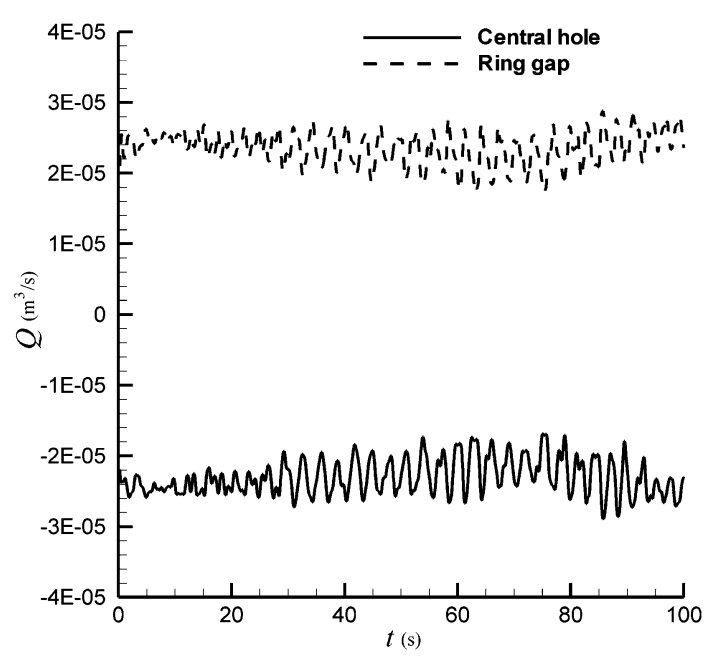

central hole, and the average velocity is about $0.06 \mathrm{~m} / \mathrm{s}$ in the gap ring (Fig. 7). The volumetric flow rate of the solvent is about $2.5 \times 10^{-5} \mathrm{~m}^{3} / \mathrm{s}$ in the central hole (Fig. 10). The volumetric flow rate though the central hole in the system with inner diameter of $10 \mathrm{~cm}$ increases 60 times compared with that in the system with inner diameter of $2.22 \mathrm{~cm}$.

\section{Conclusions}

Heat and mass transfer in an ammonothermal growth system with forward solubility were modeled. The temperature gradients across the baffle are large, which may cause some depositions of $\mathrm{GaN}$ near the baffle. The baffle opening is selected to achieve a certain temperature difference across the baffle, and thus certain supersaturation in the growth zone of the autoclave. The effects of system size and baffle opening on the flow pattern and reagent transport have been studied. For the system with inner diameter of $2.22 \mathrm{~cm}$ and baffle opening of $10 \%$, the fluid flow around the baffle is oscillatory, and goes upward in the central hole and downward through the ring gap. The constraint for continued growth of $\mathrm{GaN}$ is the growth kinetics and the total area of the seed surfaces.

For the system with inner diameter of $4.44 \mathrm{~cm}$ and baffle opening of $10 \%$, the flow around the baffle is cyclic. The fluid flow goes upward in the ring gap and downward in the central hole. For the system with inner diameter of $10 \mathrm{~cm}$ and baffle opening of $10 \%$, the flow around the baffle is cyclic due to the equal areas of the central hole and ring gap. With the increase of the inner diameter, the volumetric flow rate through the central hole increases rapidly. The nutrient transport will not be a constraint if the baffle opening is kept at $10 \%$ when scaling up the growth system. For charge particle size of $1 \mathrm{~mm}$, the low supersaturation in the growth zone can reduce depositions on the baffle and the sidewall of the autoclave. 
Acknowledgments This project is supported by the National Natural Science Foundation of China (50776098, 10972226).

\section{References}

1. T. Hashimoto, K. Fujito, M. Saito, J.S. Speck, S. Nakamura, Jpn. J. Appl. Phys. 44, 1570 (2005)

2. T. Hashimoto, K. Fujito, B.A. Haskell, P.T. Fini, J.S. Speck, S. Nakamura, J. Cryst. Growth 275, 525 (2005)

3. T. Hashimoto, M. Saito, K. Fujito, F. Wu, J.S. Speck, S. Nakamura, J. Cryst. Growth 305, 311 (2007)

4. M.P. D’Evelyn, H.C. Hong, D.-S. Park, H. Lu, E. Kaminsky, R.R. Melkote, P. Perlin, M. Lesczynski, S. Porowski, R.J. Molnar, J. Cryst. Growth 300, 11 (2007)

5. R. Dwilinki, R. Doradzinski, J. Garczynski, L.P. Sierzputowski, A. Puchalski, Y. Kanbara, K. Yagi, H. Minakuchi, H. Hayashi, J. Cryst. Growth 310, 3911 (2008)

6. R. Dwilinski, R. Doradzinski, J. Garczynski, L.P. Sierzputowski, A. Puchalski, Y. Kanbara, K. Yagi, H. Minakuchi, H. Hayashi, J. Cryst. Growth 311, 3015 (2009)

7. T. Fukuda, D. Ehrentraut, J. Cryst. Growth 305, 304 (2007)

8. D. Ehrentraut, Y. Kagamitani, C. Yokoyama, T. Fukuda, J. Cryst. Growth 310, 891 (2008)

9. K. Fujii, G. Fujimoto, T. Goto, T. Yao, Y. Kagamitani, N. Hoshino, D. Ehrentraut, T. Fukuda, J. Cryst. Growth 310, 896 (2008)

10. D. Ehrentraut, Y. Kagamitani, T. Fukuda, F. Orito, S. Kawabata, K. Katano, S. Terada, J. Cryst. Growth 310, 3902 (2008)

11. Q.S. Chen, V. Prasad, W.R. Hu, J. Cryst. Growth 258, 181 (2003)

12. Q.S. Chen, S. Pendurti, V. Prasad, J. Cryst. Growth 266, 271 (2004)

13. Q.S. Chen, S. Pendurti, V. Prasad, J. Mater. Sci. 41, 1409 (2006)

14. M. Carr, J. Fluid Mech. 509, 305 (2004)

15. V. Prasad, in Convective Heat and Mass Transfer in Porous Media, eds. by S. Kakaç et al. Convective Flow Interaction and Heat Transfer Between Fluid and Porous Layers. (Kluwer, Netherlands, 1991), p. 563 